Bundesgesundheitsbl 2019 -62:1144-1158 https://doi.org/10.1007/s00103-019-02988-w c) Springer-Verlag GmbH Deutschland, ein Teil von Springer Nature 2019

\section{Einleitung}

Gemäß $\$ \$ 16$ und 19 TFG sind Rückverfolgungsverfahren durchzuführen. Die Überprüfung des Verdachts einer Infektion bei einer spendenden Person und/oder der Möglichkeit einer Infektionsübertragung durch Blutprodukte richten sich nach dem Stand der wissenschaftlichen Erkenntnis. Dieser Notwendigkeit wurde bislang mit den Voten 34, 35 und 42 des Arbeitskreises Blut entsprochen. Die Einführung neuer Testverfahren machte eine Neufassung des Votums erforderlich.

Das Votum weist ausdrücklich die Verfahrensweisen für Infektionen mit dem Humanes Immunschwäche-Virus (HIV), dem Hepatitis-C-Virus (HCV), dem Hepatitis-B-Virus (HBV) und dem Hepatitis-E-Virus (HEV) aus. Es kann analog auch bei anderen Infektionen mit potentiell schwerwiegendem Verlauf angewendet werden.

Für die Vorgehensweise bei Variante Creutzfeldt-Jakob-Krankheit (vCJK) im Zusammenhang mit Blut, Plasma und Blutprodukten wurde ein separates Votum verabschiedet (V 46).

Das Votum gliedert sich in einen ersten Teil, in dem die Rückverfolgungsverfahren erläutert werden. In den Anhängen $A, B, C$ und $D$ werden die Untersuchungsabläufe für HIV-, HBV-, HCV- und HEVInfektionen bei der spendenden Person schematisch dargestellt und Testsensitivitäten festgelegt.

Ergänzt wird das Votum durch den Anhang E, in dem die Kriterien für die

Mitteilungen des Arbeitskreises Blut des Bundesministeriums für Gesundheit

\title{
Verfahren zur Rückverfolgung (Look Back) (gemäß § 19 Transfusionsgesetz)
}

\author{
Bei der 87. Sitzung des Arbeitskreises Blut am \\ 14.05.2019 wurde folgendes Votum (V 47) \\ verabschiedet
}

Bewertung eines Verdachts einer Infektionsübertragung durch Blutprodukte nach Abschluss des Rückverfolgungsverfahrens ausgeführt werden.

Dieses Votum ersetzt die Voten 34, 35 und 42 des Arbeitskreises Blut.

\section{Verfahren zur Rückverfolgung}

Im Verfahren zur Rückverfolgung werden die Schritte zusammengestellt, die zu beachten sind, wenn

a) bei einer spendenden Person eine Infektion mit HIV, mit Hepatitis-Viren oder anderen Erregern, die zu schwerwiegenden Krankheitsverläufen führen können, festgestellt wurde. Als Infektion ist auch der spezifische Antikörpernachweis ohne Erregernachweis zu verstehen.

b) bei einer mit Blutprodukten behandelten Person eine Infektion, insbesondere mit HIV, HBV, HCV oder HEV festgestellt wird und der begründete Verdacht besteht, dass sie durch Blutprodukte verursacht wurde,

c) nachträglich festgestellt wird, dass die zur Freigabe genutzten Testergebnisse für Infektionsmarker falsch negativ waren, deshalb zu falschen Ergebnissen geführt haben könnten und der begründete Verdacht besteht, dass das freigegebene Blutprodukt Infektionserreger übertragen könnte.

Durch das Verfahren sollen Infektionsübertragungen durch noch nicht angewendete Blutprodukte verhindert und eventuell stattgefundene Infektionen durch Blutprodukte erkannt werden. Die dafür vorgesehenen Meldewege und Meldeverpflichtungen sind in den Abschnitten 4 bis 7 dargestellt. ${ }^{1}$

Die spendende Person soll bei bestätigtem Infektionsverdacht eingehend aufgeklärt und beraten werden, insbesondere über Therapiemöglichkeiten.

Die im Rahmen des Verfahrens zur Rückverfolgung durchgeführten Maßnahmen sind gemäß $\$ 19$ TFG für Zwecke weiterer Rückverfolgungsverfahren und der Risikoerfassung nach dem Arzneimittelgesetz zu dokumentieren.

\section{Auffällige Untersuchungsergebnisse}

\subsection{Verdacht einer Infektion bei der spendenden Person}

1.1.1 Der Verdacht einer Infektion bei der spendenden Person ergibt sich aus wiederholt positiven bzw. reaktiven Ergebnissen an Untersuchungsproben, die anlässlich der Spende oder zur Freigabe von Plasma zur Transfusion aus der Quarantänelagerung abgenommen wurden.

Auch die Ergebnisse von Untersuchungen an Nachuntersuchungsproben (NUP) oder auf spezielle Anforderung gewonne-

1 Für Plasma zur Fraktionierung gelten (darüber hinaus) die Anforderungen gemäß „Guideline on plasma-derived medical products" (EMA/CHMP/706271/2010) 
ne Untersuchungsproben können den Verdacht einer Infektion auslösen, z.B. infolge anamnestischer Angaben von Symptomen, einer Erkrankung oder von Nachuntersuchungen bei nachträglichen Zweifeln an der Validität eines verwendeten Testsystems.

1.1.2 Die Infektion bei der spendenden Person ist bestätigt, wenn eine Probe mit wiederholt reaktivem und/oder positivem Screeningtest in ergänzenden Tests bestätigt positiv reagiert oder durch weiterführende Untersuchungen der initiale $\mathrm{Be}$ fund als spezifisch angesehen wird. Diese Untersuchungen können grundsätzlich abschließend aus der initialen Untersuchungsprobe durchgeführt werden.

1.1.3 Liegt ein unbestimmtes Ergebnis vor, so ist eine Bewertung anhand der Untersuchung der 2. Blutprobe vorzunehmen. Ergibt sich auch durch die Untersuchung der 2. Blutprobe ein unbestimmtes Ergebnis, so besteht kein Infektionsverdacht. In diesen Fällen muss das Rückverfolgungsverfahren nicht fortgesetzt werden.

\subsection{Verdacht einer Infektion bei einer mit Blutprodukten behandelten Person}

1.2.1 Eine Infektion bei einer mit Blutprodukten behandelten Person wird gemäß den gültigen nationalen bzw. internationalen Leitlinien ${ }^{2,3}$ zur Diagnostik nachgewiesen. Der schriftliche Befund ist an die betroffene Blutspendeeinrichtung weiterzuleiten. Weitere Meldewege und Meldeverpflichtungen sind in den Abschnitten 4 bis 7 dargestellt.

1.2.2 Der begründete Verdacht der Übertragung einer Infektion durch Blutprodukte ergibt sich bei einer mit Blutprodukten behandelten Person, wenn eine Infektion nachgewiesen ist und ernst $\mathrm{zu}$

\footnotetext{
2 AWMF; Arbeitsgemeinschaft der Wissenschaftlichen Medizinischen Fachgesellschaften e.V. (https://www.awmf.org/leitlinien/aktuelleleitlinien.html).

3 Empfehlungen der Europäischen Vereinigung für das Studium der Leber (EASL, https://easl. eu/wp-content/uploads/2018/10/EASL-CPGhepatitis-E-virus-infection.pdf).
}

nehmende Anhaltspunkte (z. B. vor Applikation bekannte negative Serologie-/ NAT-Ergebnisse; Serokonversion, Virusnachweis mit oder ohne klinische Symptome in zeitlichem Zusammenhang zur Transfusion) bestehen, dass die Infektion durch verabreichte Blutprodukte übertragen wurde (Anhang E).

\section{Untersuchungen}

\section{A Untersuchungen an initial auffälligen Proben der spendenden Person}

2.1 Ergibt die Testung von Untersuchungsproben ein wiederholt reaktives und/oder positives Ergebnis, so ist zur weiteren Abklärung des Testergebnisses an derselben Untersuchungsprobe der für dieses spezifische Virus empfohlene Untersuchungsgang unverzüglich durchzuführen (Anhänge A2, B2, C2, D).

2.2 Werden bei den zusätzlich durchgeführten Testungen nur negative Ergebnisse erhalten, so wird das in der Ersttestung (Screeningtest) erhaltene wiederholt reaktive und/oder positive Testergebnis als nicht bestätigt eingestuft.

2.3 Bei bestätigter Infektion oder unbestimmtem Ergebnis gemäß der Anhänge A2, B2, C2 bzw. D sind die betroffenen spendenden Personen eingehend aufzuklären und zu beraten.

\section{B Untersuchungen an der speziell angeforderten zweiten Blutprobe}

2.4 Ist bei der Labordiagnostik zur Feststellung des Infektionsstatus gemäß Anhang A2 und C2 das Ergebnis unbestimmt, so soll innerhalb von 7 bis 21 Tagen nach der ursprünglichen Blutabnahme eine zweite Blutprobe gewonnen werden. Eine Frist von wenigstens 7 Tagen ist einzuhalten, um eine beginnende Serokonversion deutlich werden zu lassen.

Die Testungen an der zweiten Blutprobe sind entsprechend den Untersuchungsschemata (Anhang A3, C3) durch die Spendeeinrichtung oder im Auftrag der Spendeeinrichtung durchzuführen.

\section{Nachuntersuchungsproben} (NUP)

2.5 Anlässlich jeder Spende zur Herstellung von Blutkomponenten zur allogenen Transfusion sollen mindestens $1,5 \mathrm{ml} \mathrm{Se}$ rum/Plasma abgetrennt und bei $\leq-30^{\circ} \mathrm{C}$ als NUP aufbewahrt werden. Diese NUP sollen in mindestens zwei Aliquoten in verschließbaren Behältnissen gelagert sein. Das Volumen der NUP muss so gewählt sein, dass eine Nachtestung mittels NAT sichergestellt ist.

2.6 NUP sind ein Jahr über den Ablauf des Verfalldatums des letzten Produkts der Spende hinaus, mindestens jedoch 3 Jahre aufzubewahren.

\subsection{Die Analyse von NUP dient}

- der Erhebung zusätzlicher Hinweise auf Infektiosität im Rahmen der Rückverfolgung;

- der Abklärung eventueller retrospektiv bekannt gewordener Probleme bei der Testung;

- im Falle des Nachweises eines Virusgenoms der Identitätsprüfung von Genomsequenzen bei der spendenden Person und der mit Blutprodukten behandelten Person;

- der Untersuchung auf Krankheitserreger außerhalb der Routinediagnostik im Rahmen der Rückverfolgung.

2.8 Wird auf Grund der Ergebnisse der Testungen gemäß 2.3, bzw. bei unbestimmtem Testergebnis gemäß 2.4, das Rückverfolgungsverfahren zur Aufklärung der potenziellen Infektiosität von Vorspenden notwendig, sind die vorhandenen nach 3.3 und 3.4 identifizierten NUP mittels einer sensitiven Einzelspenden-NAT auf den die Rückverfolgung auslösenden Erreger zu testen. Die analytische Sensitivität dieser Testungen ist entsprechend der Vorgaben in den Anhängen A2, B2, C2 und D zu wählen.

2.9 Wird bei einer mit Blutprodukten behandelten Person eine Infektion nachgewiesen, kann der Vergleich der Virus-Genomsequenzen aus Blut der spendenden Person und des Bluts einer mit Blutprodukten behandelten Person die Ursäch- 
lichkeit der verabreichten Blutprodukte belegen oder ggf. ausschließen.

\section{Sofortmaßnahmen und Einleitung des Rückverfolgungsverfahrens}

Der Entscheidungsgang ist im Anhang A1, B1, C1 bzw. D schematisch dargestellt.

3.1 Ergibt sich bei einer spendenden Person der Verdacht einer Infektion (1.1), ist hinsichtlich der Sperrung von Spende bzw. spendender Person gemäß dem Anhang A1, B1, C1 bzw. D zu verfahren.

3.2 Bei bestätigter Infektion oder unbestimmtem Ergebnis gemäß der Anhänge A2, B2, C2 bzw. D sind die vorausgehenden Spenden unverzüglich zu ermitteln. Damit ist das Rückverfolgungsverfahren eingeleitet.

3.3 Der Betrachtungszeitraum für die Ermittlung des Stichtages beträgt 3 Jahre für HIV, HBV und HCV und 6 Monate für HEV vor dem Entnahmedatum der Spende oder Blutprobe, deren Untersuchungsergebnis den Verdacht einer Infektion (1.1) ausgelöst hat.

3.4 Für die Ermittlung von Vorspenden gilt als Stichtag der Entnahmetag der Untersuchungsprobe der spendenden Person, in der das letzte Mal im Screening ein für den Erreger negatives Ergebnis erhoben wurde bzw. von denen für HEV kein Screening-Testergebnis vorliegt. Wird bei der Nachuntersuchung der Stichtagsspende ein positives Ergebnis festgestellt, wird der Stichtag neu festgelegt.

\section{HIV, HCV, HEV}

Alle Spenden im Zeitraum von 12 Wochen vor der Stichtagsprobe werden identifiziert. Das Rückverfolgungsverfahren kann abgeschlossen werden, sobald in der NUP der Stichtagsspende ein negatives Einzelspenden-NAT (ID-NAT) Ergebnis für HIV, HCV bzw. HEV vorliegt.

\section{HBV}

Für HBV werden die Stichtagsspende und alle dem Stichtag 16 Wochen vorausgehenden Spenden identifiziert und getestet. Das Rückverfolgungsverfahren kann abgeschlossen werden, wenn alle NUP im neu festgelegten Untersuchungszeitraum ein negatives Ergebnis aufweisen. ${ }^{4}$

3.5 Alles in der Spendeeinrichtung noch vorhandene Blut bzw. alle noch vorhandenen Bestandteile aus Blut werden ausgesondert und sichergestellt.

3.6 Einrichtungen der Krankenversorgung/ärztliche Personen, an die Blutprodukte aus gemäß 3.4 identifizierten Spenden geliefert wurden, werden unverzüglich benachrichtigt, soweit Blutprodukte noch in der Laufzeit sind. Sie sondern noch vorhandene Blutprodukte aus und stellen diese sicher.

3.7 Wurde Plasma aus gemäß 3.4 identifizierten Spenden an ein Plasma verarbeitendes Unternehmen geliefert, wird das Plasma verarbeitende Unternehmen vereinbarungsgemäß über identifizierte Vorspenden informiert, ausgenommen hiervon sind isoliert Anti-HBc reaktive Plasmen.

3.8 Ergeben sich nachträglich Hinweise, dass die zur Freigabe einer Spende von Blut oder Bestandteilen aus Blut vorgenommenen Testungen auf Virusmarker möglicherweise falsch negativ waren, ist eine Risikobewertung ggf. mit der zuständigen Behörde durchzuführen. Entsprechend dem Ergebnis sind geeignete Sofortmaßnahmen einzuleiten.

\section{Meldesysteme und Meldeverpflichtungen bei Verfahren zur Rückverfolgung}

4.1 Die Spendeeinrichtung unterhält ein Meldesystem, das bei bestätigter Infektion oder unbestimmtem Ergebnis gemäß der Anhänge A2, B2, C2 bzw. D der spendenden Person die unverzügliche Benachrichtigung von Einrichtungen der Krankenversorgung/ärztlichen Personen im Rahmen der Sofortmaßnahmen (3.6) sicherstellt. Zum gleichen Zweck ist ein Meldesystem zur gegenseitigen Unterrichtung zwischen der Spendeeinrichtung und

\footnotetext{
${ }^{4}$ Für Plasma zur Fraktionierung 6 Monate gemäß ${ }_{\text {"Guideline on plasma-derived medical }}$ products" (EMA/CHMP/706271/2010).
}

dem Plasma verarbeitenden Unternehmen einzurichten (3.7).

4.2 Ergibt sich bei einer mit Blutprodukten behandelten Person der begründete Verdacht einer transfusionsbedingten Infektion (1.2), bestehen die Unterrichtungspflichten nach $\$ 16$ TFG. Ebenso sind die Dokumentations- und Anzeigepflichten, die sich aus dem AMG ergeben, zu beachten (vgl. z. B. \$63i Absatz 2 AMG, Meldung von schwerwiegenden unerwünschten Reaktionen an die zuständige Bundesoberbehörde innerhalb von $15 \mathrm{Ta}$ gen). Hierzu werden Meldeformulare von der zuständigen Bundesoberbehörde zur Verfügung gestellt. Die Meldungen an die Arzneimittelkommission der deutschen Ärzteschaft bleiben unberührt.

4.3 Die unverzügliche Meldung der Einleitung eines Rückverfolgungsverfahrens an die zuständigen Behörden regelt sich nach $\$ 19$ (1) TFG. Für die von der spendenden Person ausgehenden Rückverfolgungsverfahren stehen eine Erläuterung sowie für diese Meldung empfohlene Meldebögen der Zentralstelle der Länder für Gesundheitsschutz (ZLG) und des PaulEhrlich-Instituts (PEI) im Internet auf der Homepage des PEI zum Download bereit.

\section{Unterrichtung der spendenden Person über den festgestellten Infektionsstatus}

5.1 Ergibt sich aus der Analyse von Untersuchungsproben ein bestätigt positives Ergebnis (Anhang A2, B2, C2, D) wird die spendende Person durch eine von der Spendeeinrichtung benannte ärztliche Person und/oder der von ihr angegebenen ärztlichen Person ihres Vertrauens unverzüglich informiert. Diese Information, Aufklärung und Beratung ist in jedem Einzelfall nach Art und Umfang festzulegen und zu dokumentieren.

5.2 Bei wiederholt unbestimmten Untersuchungsergebnissen (siehe Anhang A2, A3, C2, C3) sollte die Information und Beratung der spendenden Person analog 5.1 erst nach Vorliegen der Ergebnisse der 2. Blutprobe vorgenommen werden. Die Entscheidung für eine zeitweise oder dauerhafte Sperrung der spendenden Per- 
son obliegt der Spendeeinrichtung (siehe Anhang A1, C1).

5.3 Ergibt die HBV-Labordiagnostik bei der spendenden Person eine spezifische Anti-HBc-Reaktivität (siehe Anhang B2), erfolgt eine Information und Beratung der spendenden Person analog 5.1.

5.4 Kann innerhalb von 5 Wochen nach dem Entnahmedatum der Spende der Infektionsstatus nicht sicher festgestellt werden, ist die spendende Person analog 5.1 $\mathrm{zu}$ informieren und eine Beratungsmöglichkeit anzubieten.

\section{Von der spendenden Person ausgehendes Rückverfolgungsverfahren in Spendeeinrichtungen}

6.1 Ergibt sich bei einer spendenden Person eine bestätigte Infektion oder ein unbestimmtes Ergebnis gemäß der Anhänge A2, B2, C2 bzw. D, so ist das Rückverfolgungsverfahren zur Aufklärung der potenziellen Infektiosität von vorhandenen oder schon verabreichten Blutprodukten aus Vorspenden durchzuführen.

6.2 Von nach 3.3 und 3.4 identifizierten Untersuchungsproben oder Spenden werden die früher erhobenen Untersuchungsergebnisse anhand der Dokumentation überprüft.

6.3 NUP und in der Einrichtung verfügbare zusätzliche Untersuchungsproben von Entnahmen, die im nach 3.4 definierten Zeitraum auch unabhängig von Spenden gewonnen wurden, werden ermittelt. Die Auswahl der zu testenden Proben ist unter 3.4 beschrieben. Die Sensitivität der nach 2.8 vorgeschriebenen ID-NAT ist in den Anhängen A2, B2, C2 und D festgelegt.

6.4 Werden bei der Untersuchung dieser Proben negative Ergebnisse erhalten, wird das Rückverfolgungsverfahren beendet.

6.5 Werden bei der Untersuchung dieser Probe(n) Ergebnisse erhalten, die darauf hinweisen, dass die spendende Person schon zum Zeitpunkt der Probennahme infektiös gewesen sein kann, wird der
Stichtag nach 3.4 neu festgelegt und analog verfahren.

6.6 Ist eine Untersuchung der NUP nicht möglich, soll das Rückverfolgungsverfahren mit den transfundierenden Einrichtungen fortgeführt werden. Hierzu werden die Personen identifiziert, die Blutprodukte aus der letzten im Screening negativen Vorspende (Stichtagsspende) und aus den vorangegangenen Spenden im Zeitraum

- bei HIV: 12 Wochen

- bei HCV: 12 Wochen

- bei HBV: 16 Wochen

erhalten haben. Den Einrichtungen wird die diagnostische Abklärung einer möglichen Transmission gemäß den gültigen nationalen bzw. internationalen Leitlinien zur Diagnostik empfohlen. Wenn die Personen, die Blutkomponenten dieser Spenden erhalten haben, nicht verfügbar sind, ist das Rückverfolgungsverfahren beendet.

Für HEV gilt das Rückverfolgungsverfahren ebenfalls als beendet, sofern eine Untersuchung der NUP der Stichtagsspende und der vorausgegangenen Spenden im Zeitraum von 12 Wochen nicht mehr möglich ist.

6.7 Wurden nach 6.5 zusätzliche Spenden identifiziert, werden evtl. in der Spendeeinrichtung noch vorhandene Blut oder Bestandteile aus Blut ebenfalls sichergestellt und ausgesondert. Die Meldeverpflichtungen gelten gemäß 4.1.

6.8 Einrichtungen der Krankenversorgung/ärztliche Personen, an die Blutprodukte aus identifizierten Spenden geliefert wurden, werden über positive Ergebnisse aus der NUP unverzüglich unterrichtet.

6.9 Die Einrichtungen der Krankenversorgung/ärztliche Personen ermitteln die Personen, die Blutprodukte aus identifizierten Spenden erhalten haben und leiten gemäß\$19 (1) Satz 6-8 TFG entsprechende Maßnahmen ein, wenn ein begründeter Verdacht einer transfusionsbedingten Infektion besteht bzw. nicht ausgeschlossen werden konnte, da z. B. eine Untersuchung der NUP nicht möglich ist.

6.10 Ergibt die diagnostische Abklärung Ergebnisse, die eine transfusionsbedingte
Infektion ausschließen (Anhang E) bzw. ist eine diagnostische Abklärung nicht möglich, beendet die Spendeeinrichtung das Rückverfolgungsverfahren.

6.11 Ergibt die diagnostische Abklärung bei den Personen, die Blutprodukte erhalten haben, den Verdacht einer transfusionsbedingten Infektion, versucht die Spendeeinrichtung durch geeignete Untersuchungen (z.B. vergleichende Genomanalyse), die Kausalität abzuklären (Anhang E).

6.12 Die Einrichtung der Krankenversorgung ist dafür verantwortlich, dass die Personen, die diese Blutprodukt erhalten haben, über das Ergebnis der Untersuchung unterrichtet und eingehend beraten werden.

\section{Von mit Blutprodukten behandelten Personen ausgehendes Rückverfolgungsverfahren}

\section{A Einleitung des \\ Rückverfolgungsverfahrens}

7.1 Ergibt sich bei einer Person, die Blutprodukte erhalten hat, der Nachweis einer Infektion und daraus der begründete Verdacht einer Übertragung durch Blutprodukte (1.2), wird von der behandelnden ärztlichen Person bzw. der Einrichtung zur Krankenversorgung gemäß \$19 (2) in Verbindung mit $\$ 16$ TFG unverzüglich ein Rückverfolgungsverfahren eingeleitet.

7.2 Von der behandelnden ärztlichen Person bzw. der Einrichtung der Krankenversorgung werden alle der Person verabreichten Blutprodukte und die zugeordneten pharmazeutischen Unternehmer ermittelt.

7.3 Die pharmazeutischen Unternehmen werden unverzüglich über den Infektionsverdacht und die verabreichten Blutprodukte unterrichtet. Das pharmazeutische Unternehmen führt das Rückverfolgungsverfahren fort und unterrichtet über das Ergebnis die verantwortliche ärztliche Person, die ihrerseits die Person, die Blutprodukte erhalten hat, sachgerecht unterrichtet und eingehend berät. Die Melde- 


\begin{tabular}{|c|c|}
\hline Abkürzungen & \\
\hline$A M G$ & Arzneimittelgesetz \\
\hline Analytische Sensitivität & $\begin{array}{l}\text { In diesem Votum gleichzusetzen mit Nachweisgrenze für die NAT-Tests. } \\
\text { Nach den CTS (Common Technical Specifications) die Konzentration, bei } \\
\text { der } 95 \% \text { der Ansätze reaktiv sind. }\end{array}$ \\
\hline $\begin{array}{l}\text { Blutprodukte (Begriff } \\
\text { des Transfusions- } \\
\text { gesetzes) }\end{array}$ & $\begin{array}{l}\text { Blutzubereitungen im Sinne des } \S 4 \text { Abs. } 2 \text { des Arzneimittelgesetzes, Sera } \\
\text { aus menschlichem Blut im Sinne des } \S 4 \text { Abs. } 3 \text { des Arzneimittelgesetzes } \\
\text { und Blutbestandteile, die zur Herstellung von Wirkstoffen oder } \\
\text { Arzneimitteln bestimmt sind. }\end{array}$ \\
\hline Empfangende Person & Eine Person, auf die Blutprodukte übertragen worden sind. \\
\hline$H B V$ & Hepatitis-B-Virus \\
\hline$H B C$ & Hepatitis-B-Core-Antigen \\
\hline$H B s A g$ & Hepatitis-B-Oberflächenantigen \\
\hline HCV & Hepatitis-C-Virus \\
\hline HEV & Hepatitis-E-Virus \\
\hline$H I V$ & Humanes Immunschwächevirus \\
\hline $\begin{array}{l}\text { Nachuntersuchungs- } \\
\text { proben (NUP) }\end{array}$ & $\begin{array}{l}\text { Anlässlich einer Spende von Blut oder Bestandteilen aus Blut aufbewahr- } \\
\text { te Untersuchungsproben einer spendenden Person, die im Falle eines } \\
\text { Rückverfolgungsverfahrens die Nachuntersuchung der Ausgangsspende } \\
\text { auf Infektionsmarker ermöglichen. }\end{array}$ \\
\hline NAT & Nukleinsäure-Amplifikations-Technik \\
\hline Negativ & Serologisch nicht reaktiv bzw. Genom (NAT) nicht nachweisbar \\
\hline Positiv & Genomnachweis (NAT) \\
\hline Reaktiv & Serologisch nicht negativ (Messwert > testspezifischem Cut-off) \\
\hline Spende & $\begin{array}{l}\text { Die Spende ist die bei Menschen entnommene Menge an Blut oder } \\
\text { Blutbestandteilen, die Wirkstoff oder Arzneimittel ist oder zur Herstel- } \\
\text { lung von Wirkstoffen oder Arzneimitteln und anderen Produkten zur } \\
\text { Anwendung beim Menschen bestimmt ist. }\end{array}$ \\
\hline Spendeeinrichtung & $\begin{array}{l}\text { Eine Einrichtung, die Spenden entnimmt oder deren Tätigkeit auf die } \\
\text { Entnahme von Spenden und, soweit diese zur Anwendung bestimmt } \\
\text { sind, auf deren Testung, Verarbeitung, Lagerung und das Inverkehrbrin- } \\
\text { gen von Blutprodukten gerichtet ist. }\end{array}$ \\
\hline TFG & Transfusionsgesetz \\
\hline
\end{tabular}

verpflichtungen entsprechend 4 sind $\mathrm{zu}$ beachten.

\section{B Weiteres Vorgehen bei \\ Blutprodukten}

7.4 Das pharmazeutische Unternehmen, das die verabreichten Blutprodukte hergestellt hat, veranlasst die Identifikation der zugehörigen spendenden Personen.

7.5 Eventuell vorhandene Blutprodukte aus Spenden der implizierten spendenden Person werden identifiziert und sichergestellt, sofern nicht die Kriterien unter Punkt 7.7 erfüllt sind.

7.6 Von identifizierten Spenden, aus denen andere als der infizierten Person verabreichte Blutprodukte hergestellt wurden, werden die früher erhobenen Laborbefunde an Hand der Dokumentation überprüft.

7.7 Die Spendeeinrichtung ermittelt, ob spätere Untersuchungen an der/den spendenden Person(en) eine entsprechende Infektion ausschließen oder unwahrscheinlich machen:

- falls die spendende Person 12 Wochen oder später nach der Spende im Screening Anti-HIV, HIV-1-NAT, Anti-HCV und HCV-NAT negativ war, ist die verdächtigte Spende als nicht infektiös hinsichtlich HIV und HCV zu werten;

- falls die spendende Person 16 Wochen oder später im Screening Anti$\mathrm{HBc}$ und $\mathrm{HBsAg}$ und gegebenenfalls HBV-NAT negativ war, ist die ver- dächtigte Spende als nicht infektiös hin-sichtlich HBV zu werten.

Für HEV nicht anwendbar, da eine spätere HEV-NAT Untersuchung keine sichere Aussage hinsichtlich der Infektiosität zum Stichtag erlaubt.

7.8 Liegen keine zur Abklärung beitragenden Untersuchungsergebnisse vor, wird die spendende Person unverzüglich zu einer diagnostischen Abklärung aufgefordert. Die Blutabnahme erfolgt so bald wie möglich. Liegt die implizierte Spende vom Zeitpunkt der neuerlichen Probennahme weniger als

- 12 Wochen bei HIV

- 12 Wochen bei HCV

- 16 Wochen bei HBV

zurück, muss zusätzlich zu dieser Probe eine weitere Blutprobe zu einem Zeitpunkt 12 Wochen bei HIV- und HCV-Infektion und 16 Wochen bei HBV-Infektion nach der implizierten Spende genommen werden.

Für HEV nicht anwendbar, da eine spätere HEV-NAT Untersuchung keine sichere Aussage hinsichtlich der Infektiosität zum Stichtag erlaubt.

7.9 Ist eine Infektion nach 7.7 nicht ausgeschlossen und eine zweite Blutprobe nach 7.8 nicht verfügbar, werden vorhandene NUP der identifizierten Spenden der verabreichten Blutprodukte auf den die Rückverfolgung auslösenden Erreger mittels ID-NAT untersucht.

7.10 Wird eine spendende Person identifiziert, bei der der Verdacht einer Infektion besteht, muss ein Rückverfolgungsverfahren entsprechend Kapitel 6 durchgeführt werden.

7.11 Ist eine diagnostische Abklärung bei der spendenden Person nicht möglich, wird das von der spendenden Person ausgehende Rückverfolgungsverfahren dennoch eingeleitet.

7.12 Die Beurteilung des Kausalzusammenhangs ist nach $\$ 63 \mathrm{i} A \mathrm{AM}$ vorgeschrieben und richtet sich nach dem Anhang E.

Für den Arbeitskreis Blut

Dr. R. Offergeld

Vorsitzende 


\section{Anhang}

A1:HIV

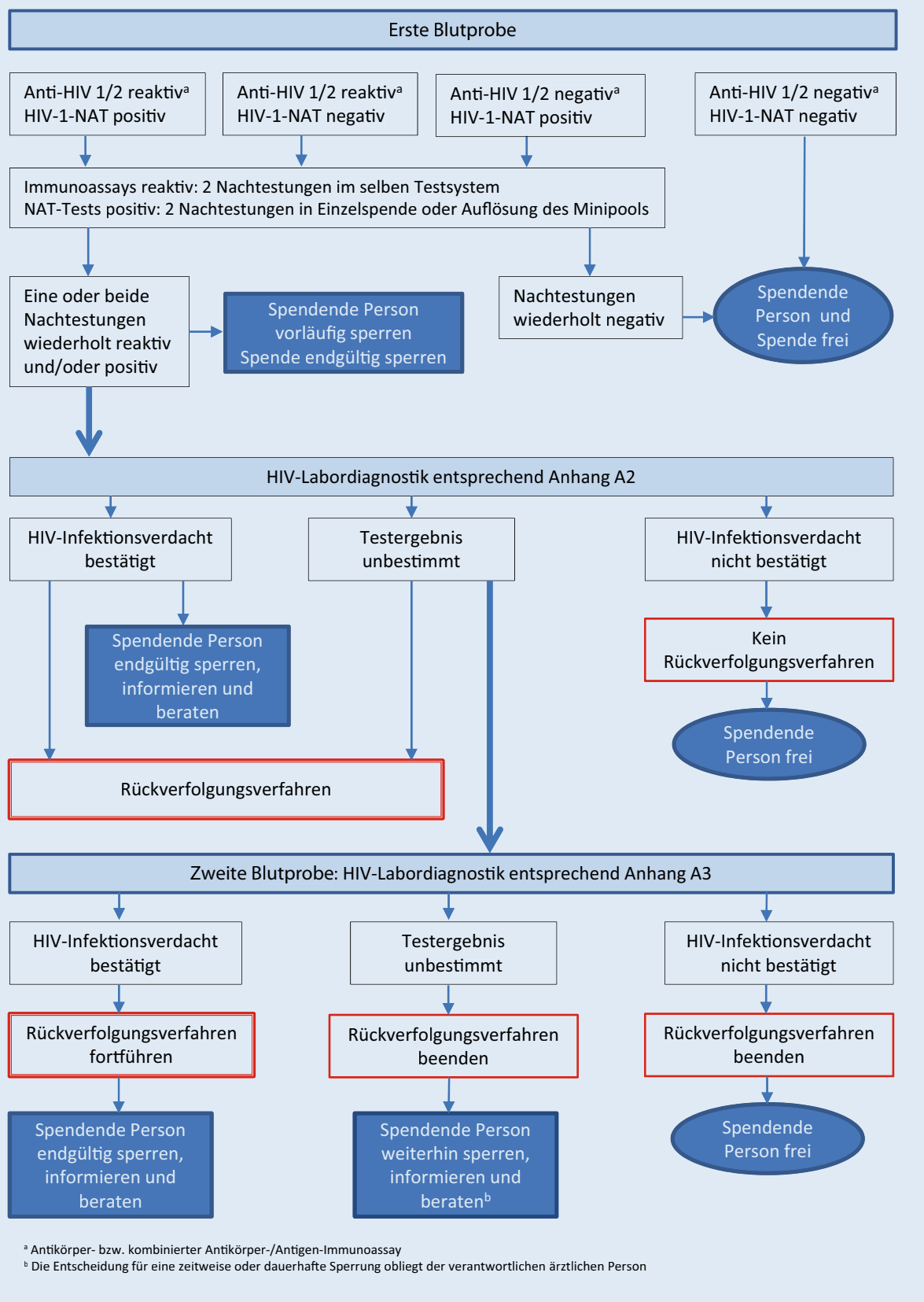


A2: HIV

Ergebnisse der gemäß Anhang A1

an der ersten Blutprobe

durchgeführten Screeningtests:

HIV 1/2-Immunoassaya ${ }^{a}$ HIV-1-NAT
Humanes Immundefizienz Virus (HIV)

Erste Blutprobe: Labordiagnostik zur Feststellung des Infektionsstatus

Anti-HIV $1 / 2^{\mathrm{a}}$ negativ

HIV-1-NAT in der Einzelspende oder

nach Auflösung des Minipools

bestätigt

wiederholt positiv

Anti-HIV $1 / 2^{a}$ wiederholt reaktiv HIV-1-NAT in der Einzelspende oder nach Auflösung des Minipools

wiederholt positiv

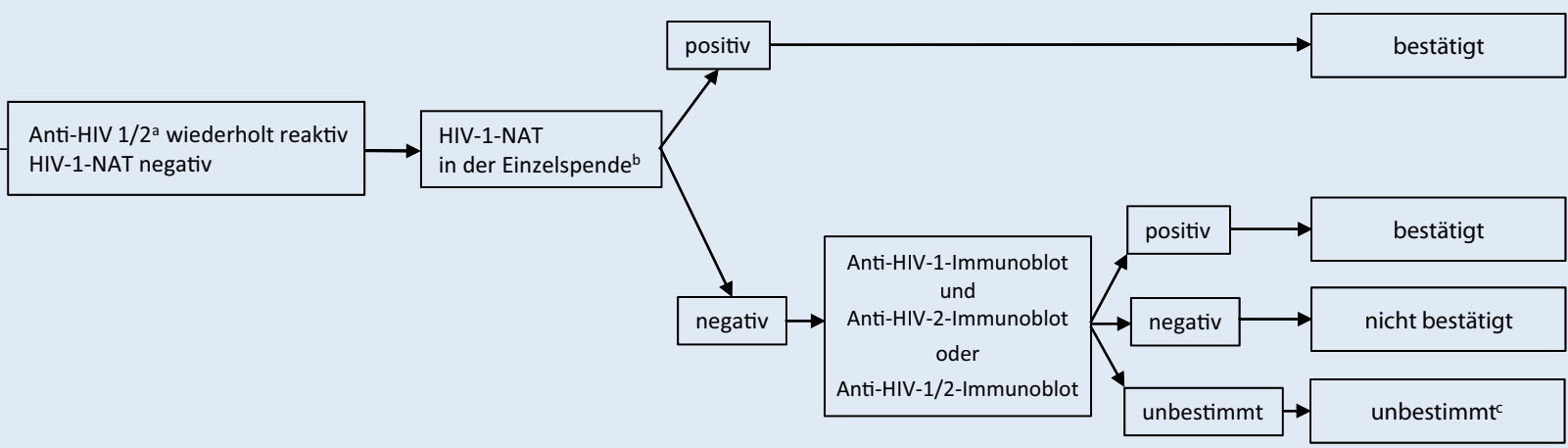

a Antikörper-bzw, kombinierter Antikörper-/Antigentest

${ }^{b}$ Analytische Sensitivität der Dual-target HIV-1 NAT: $<100 \mathrm{IU} / \mathrm{mL}$

${ }^{c}$ Der Infektionsverdacht ist aneiner zweiten Blutprobe nach Anhang $\mathrm{A} 3$ abzuklären. 
Humanes Immundefizienz Virus (HIV)

\section{Zweite Blutprobe: Labordiagnostik zur Feststellung des Infektionsstatus}

Ergebnisse der an der zweiten,

neu entnommenen Blutprobe

durchgeführten Bestätigungstests:

Anti-HIV-1/2 Immunoassay ${ }^{a}$

wiederholt negativ

iederholt reaktiv

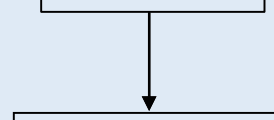

Anti-HIV-1-Immunoblot und

Anti-HIV-2-Immunoblot

oder

Anti-HIV1/2-Immunoblot

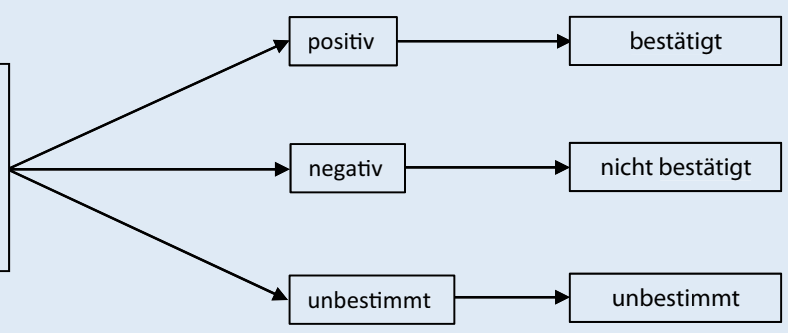




\section{Erste Blutprobe}

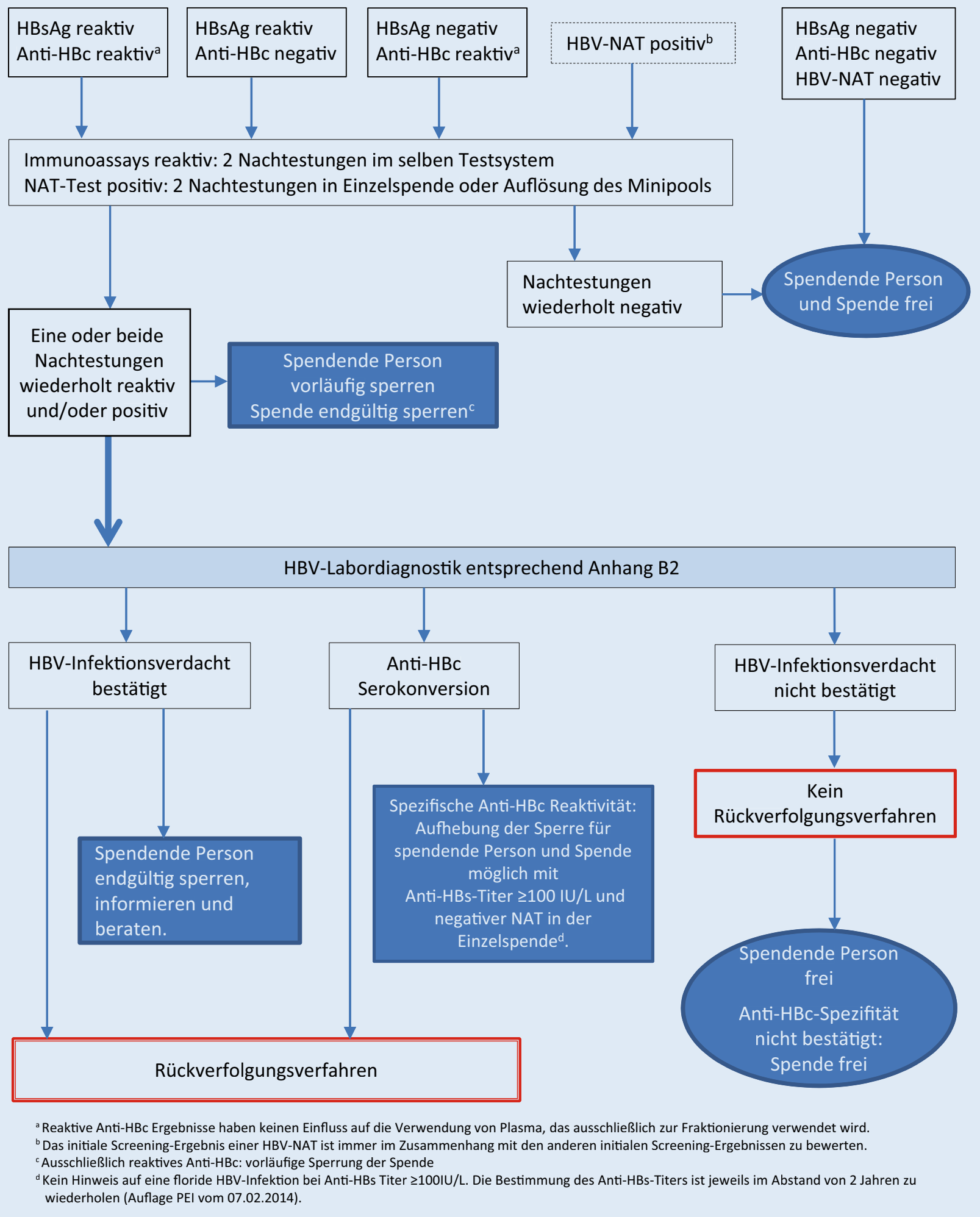




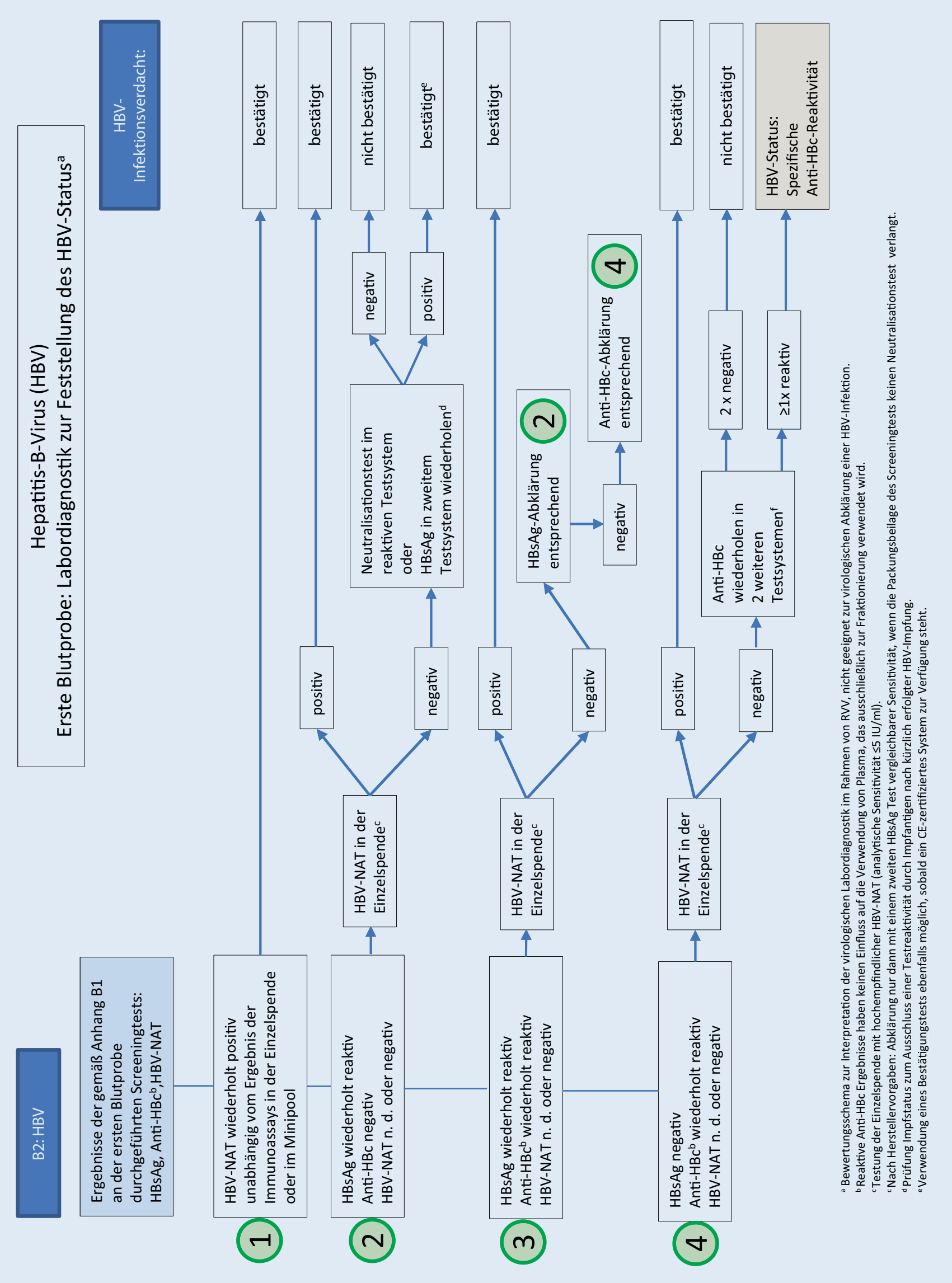




\section{C1:HCV}

Erste Blutprobe

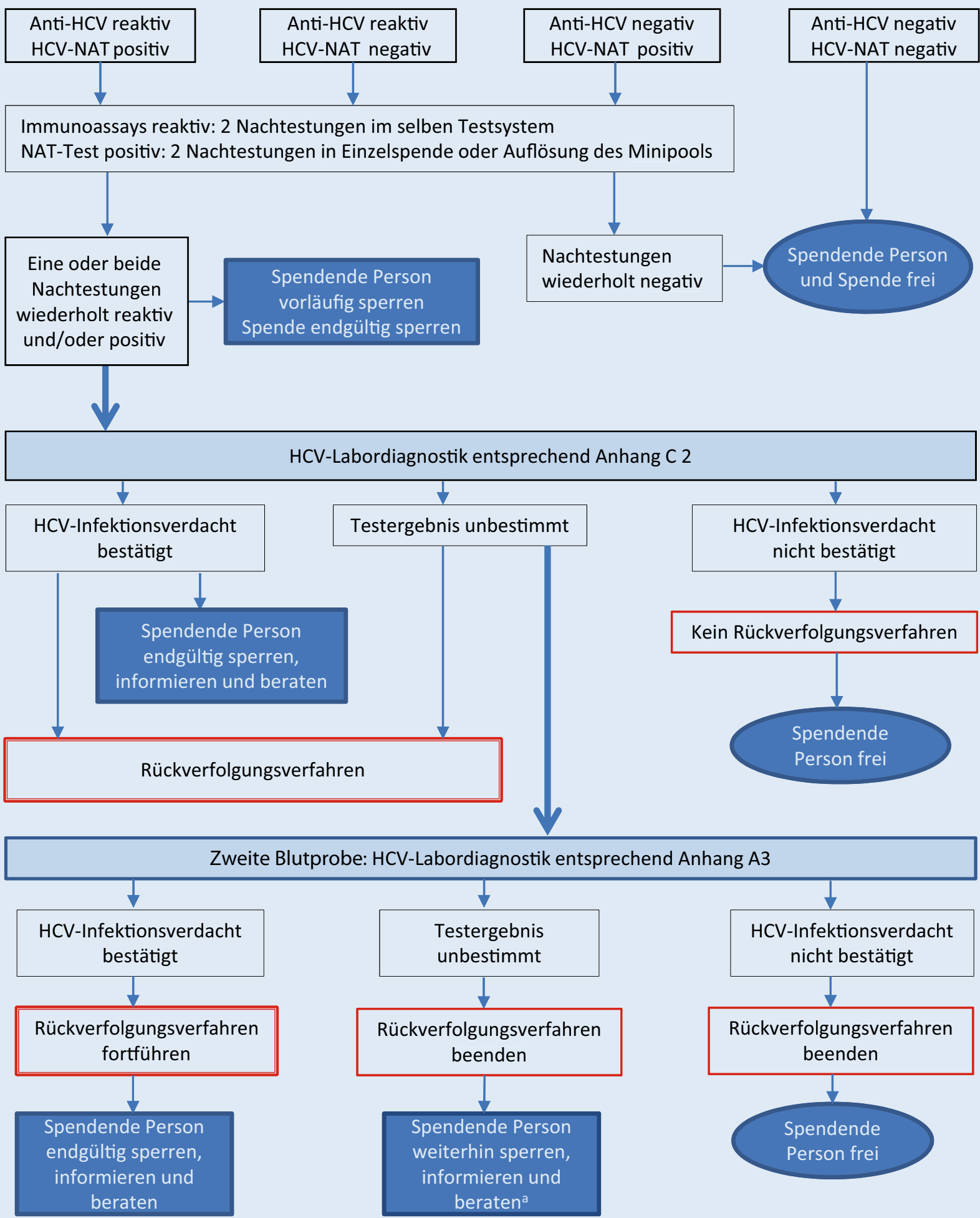

a Die Entscheidung für eine zeitweise oder dauerhafte Sperrung obliegt der verantwortlichen ärztlichen Person. 
Ergebnisse der gemäß Anhang C1

an der ersten Blutprobe

durchgeführten Screeningtests:

Anti-HCV-Immunoassay, HCV-NAT

Hepatitis-C-Virus (HCV)

Erste Blutprobe: Labordiagnostik zur Feststellung des Infektionsstatus

Anti-HCV negativ

HCV-NAT wiederholt positiv
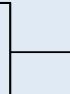

bestätigt

Anti-HCV wiederholt reaktiv

HCV-NAT wiederholt positiv
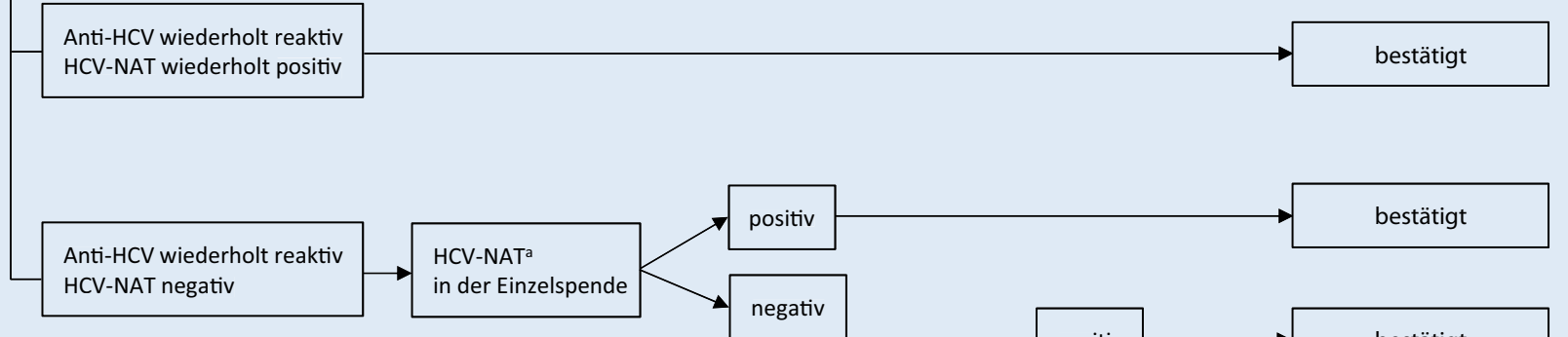

positiv

bestätigt
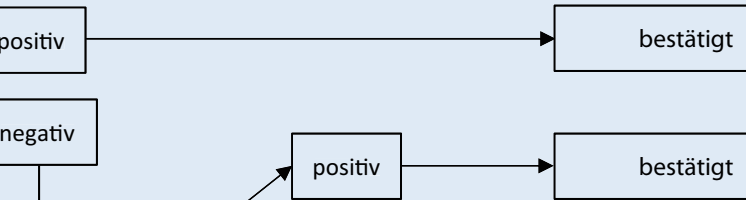

Anti-HCV Immunoblot

negativ

nicht bestätigt

unbestimmt

unbestimmt ${ }^{\mathrm{b}}$

a Testung mit einer hochempfindlichen NAT, analytische Sensitivität $<20 \mathrm{IU} / \mathrm{mL}$

${ }^{\mathrm{b}}$ Für die Sicherung der Infektion bei der spendenden Person sind zusätzliche Untersuchungen an einer zweiten Blutprobe erforderlich (siehe C 1 und C 3 ) 
Ergebnisse der an der zweiten, neu entnommenen Blutprobe durchgeführten Bestätigungstests:

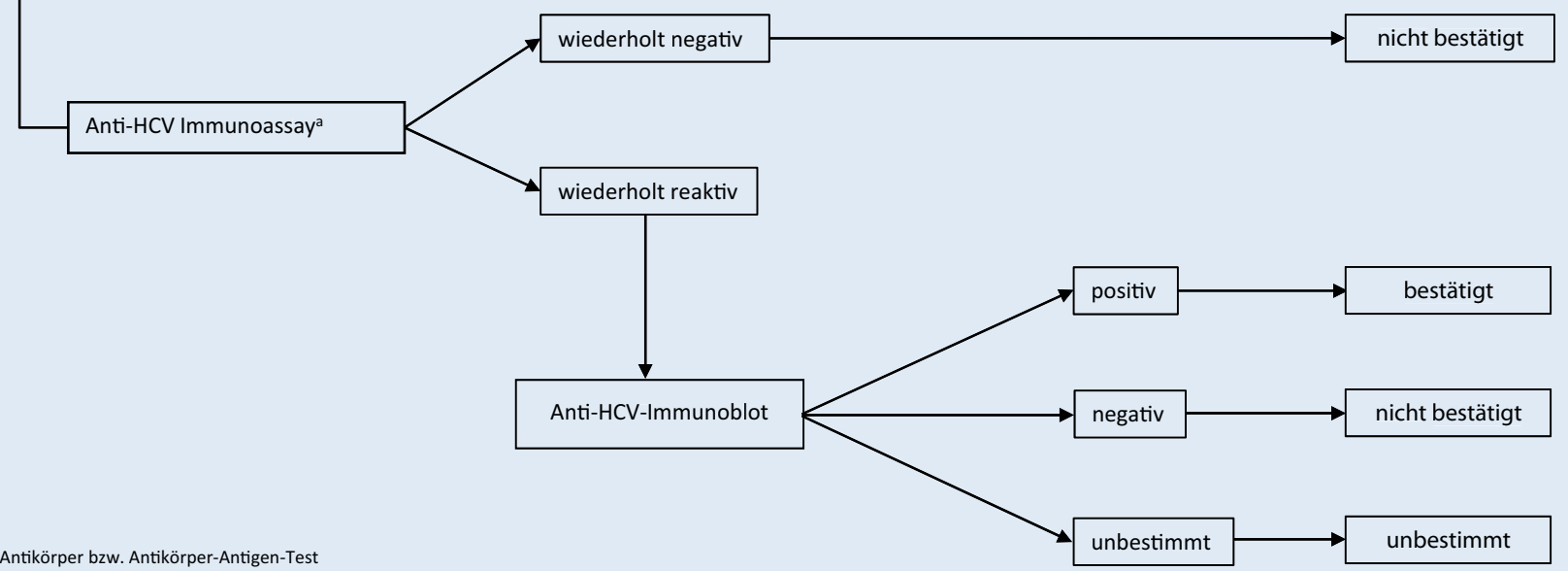

Erste Blutprobe
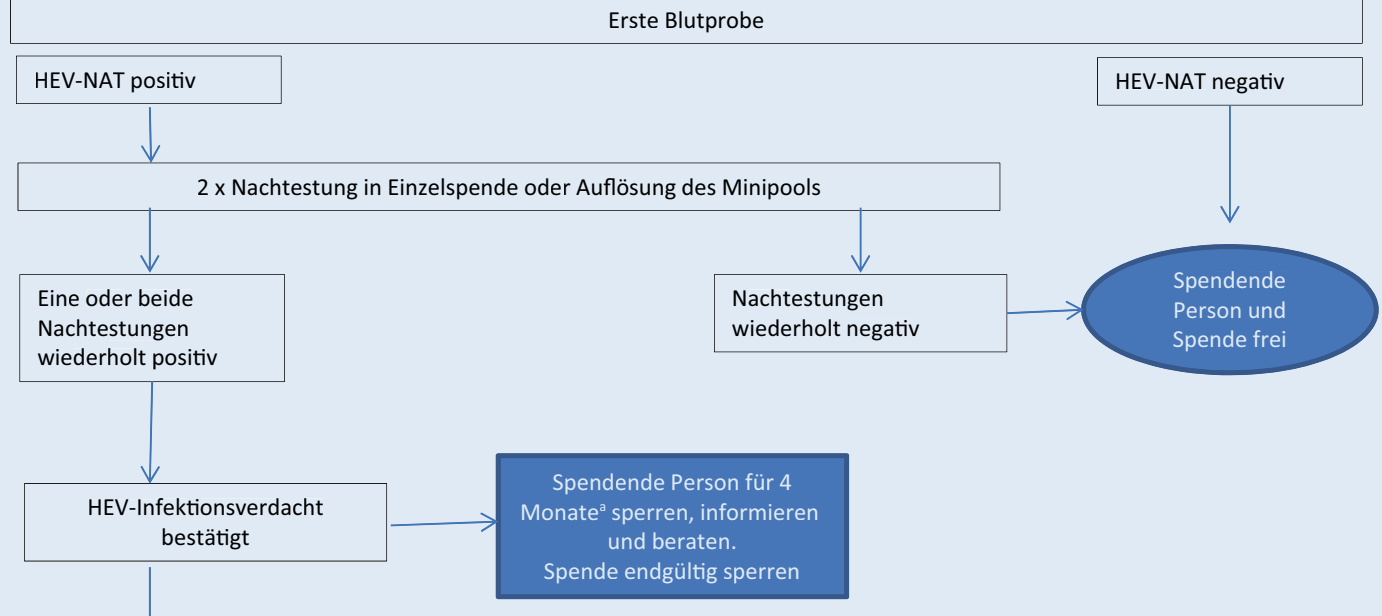

Rückverfolgungsverfahren

a Wiederzulassung zur Spende $\geq 4$ Wochen nach HEV-NAT positiver Spende ist möglich bei negativem Ergebnis einer HEV-NAT in der Einzelspende mit einer analytischen Sensitivität von mindestens $50 \mathrm{IU} / \mathrm{ml}$.

${ }^{b}$ Für die NUP ist eine Mindestsensitivität von $50 \mathrm{IU} / \mathrm{mL}$ erforderlich 


\section{Anhang $\mathrm{E}$}

Tab. 1 Zuordnungsstufen für schwerwiegende unerwünschte Reaktionen gemäß Richtlinie 2005/61/EGa; adaptiert für die Bewertung des Verdachts einer Virusübertragung durch Blutprodukte

\begin{tabular}{|c|c|}
\hline Bewertung & Zuordnungsstufen \\
\hline Sicher & $\begin{array}{l}\text { Es steht eindeutig und zweifelsfrei fest, dass die unerwünschte Reaktion auf Blut oder Blutbestandteile zurückzuführen ist: } \\
\text { Die Infektionen in der spendenden und der Blutprodukte empfangenden Person sowie ein zeitlich plausibler Zusammenhang } \\
\text { mit der Transfusion und eine eindeutige phylogenetische Sequenzhomologie beweisen, dass das Blutprodukt die Infektion in der } \\
\text { empfangenden Person verursacht hat }\end{array}$ \\
\hline Wahrscheinlich & $\begin{array}{l}\text { Es liegen deutliche Anzeichen dafür vor, dass die unerwünschte Reaktion auf Blut oder Blutbestandteile zurückzuführen ist: } \\
\text { Der zeitlich passende Zusammenhang und zusätzliche anamnestische Erkenntnisse (Infektion in spendender und empfangender } \\
\text { Person, außer Transfusion keine Übertragungswege plausibel), sprechen dafür, dass das Blutprodukt die Infektion in der empfan- } \\
\text { genden Person verursacht hat. Eine vergleichende Sequenzanalyse liegt nicht vor }\end{array}$ \\
\hline Möglich & $\begin{array}{l}\text { Es steht nicht eindeutig fest, ob die unerwünschte Reaktion auf Blut oder Blutbestandteile oder auf andere Ursachen zurückzufüh- } \\
\text { ren ist: } \\
\text { Trotz der Infektion in der spendenden Person und des zeitlichen Zusammenhangs von Transfusion und Infektion in der empfan- } \\
\text { genden Person, gibt es medizinisch plausible alternative Erklärungsmöglichkeiten, die eine Infektion durch die transfundierten } \\
\text { Blutprodukte nur als eine von mehreren Möglichkeiten erscheinen lassen. Eine vergleichende Sequenzanalyse liegt nicht vor }\end{array}$ \\
\hline Unwahrscheinlich & $\begin{array}{l}\text { Es liegen deutliche Anzeichen dafür vor, dass die unerwünschte Reaktion auf andere Ursachen als die Gabe von Blut oder Blutbe- } \\
\text { standteile zurückzuführen ist: } \\
\text { Trotz nachgewiesener Infektion in der spendenden und empfangenden Person sprechen der unklare zeitliche Zusammenhang } \\
\text { mit der Transfusion, die vorliegenden anamnestische Angaben und die fehlende relevante phylogenetische Sequenzhomologie } \\
\text { gegen die Transfusion als Ursache der Infektion }\end{array}$ \\
\hline Ausgeschlossen & $\begin{array}{l}\text { Es steht zweifelsfrei fest, dass die unerwünschte Reaktion auf andere Ursachen als die Gabe von Blut oder Blutbestandteile zurück- } \\
\text { zuführen ist: } \\
\text { Das transfundierte Blutprodukt kann als Ursache der Infektion in der empfangenden Person ausgeschlossen werden, sofern eine } \\
\text { der drei nachfolgend genannten Möglichkeiten zutrifft. } \\
\text { - keine Infektion in der spendenden Person } \\
\text { - Infektion in der spendenden Person, aber keine Infektion in der empfangenden Person in zeitlich plausiblem Zusammenhang } \\
\text { mit der Transfusion (z. B. Merkmale der Infektion im Empfänger bereits zum Zeitpunkt der Transfusion) } \\
\text { - Infektion in spendender und empfangender Person, zeitlich plausibler Zusammenhang mit der Transfusion, aber Sequenzana- } \\
\text { lyse schließt Identität des Virus in spendender und empfangender Person aus (unterschiedliche Genotypen bzw. Subtypen) }\end{array}$ \\
\hline Nicht bewertbar & $\begin{array}{l}\text { Die Daten reichen für eine Zuordnungsbewertung nicht aus: } \\
\text { Die Datenlage ist ungenügend, weil z. B. wegen unzureichenden Probenvolumens oder zu geringer Viruslast keine aussagefähi- } \\
\text { gen Daten erhoben werden können bzw. weil spendende oder empfangende Person nicht mehr verfügbar sind }\end{array}$ \\
\hline \multicolumn{2}{|c|}{$\begin{array}{l}\text { aRICHTLINIE 2005/61/EG DER KOMMISSION vom 30. September } 2005 \text { zur Durchführung der Richtlinie 2002/98/EG des Europäischen Parlaments und des Rates in Bezug } \\
\text { auf die Anforderungen an die Rückverfolgbarkeit und die Meldung ernster Zwischenfälle und ernster unerwünschter Reaktionen. Anhang II Teil B: Ernste unerwünschte } \\
\text { Reaktionen - Zuordnungsstufen }\end{array}$} \\
\hline
\end{tabular}


Tab. 2 Ausschlusskriterien für den Zusammenhang einer Virusinfektion mit einer Transfusion

\begin{tabular}{|c|c|c|c|c|}
\hline & HIV & HBV & HCV & HEV \\
\hline \multirow[t]{3}{*}{$\begin{array}{l}\text { Ursächlichkeit des } \\
\text { Blutprodukts für } \\
\text { Empfängerinfektion } \\
\text { ausgeschlossen wenn: }\end{array}$} & 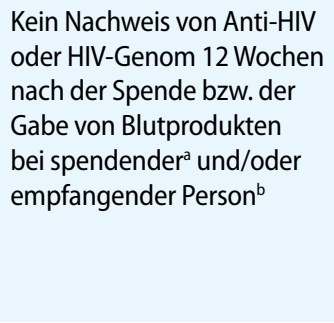 & $\begin{array}{l}\text { Kein Nachweis von HBsAg, } \\
\text { Anti-HBc oder HBV-Genom } \\
16 \text { Wochen nach der Spende } \\
\text { bzw. der Gabe von Blutpro- } \\
\text { dukten bei spendender } \\
\text { und/oder empfangender } \\
\text { Person }\end{array}$ & $\begin{array}{l}\text { Kein Nachweis von Anti-HCV } \\
\text { oder HCV-Genom } 12 \text { Wo- } \\
\text { chen nach der Spende bzw. } \\
\text { der Gabe von Blutprodukten } \\
\text { bei spendendera und/oder } \\
\text { empfangender Person }^{\text {b }}\end{array}$ & $\begin{array}{l}\text { Kein Nachweis von HEV- } \\
\text { Genom in der Nachuntersu- } \\
\text { chungsprobe der verdäch- } \\
\text { tigten Spende und/oder kein } \\
\text { Nachweis von HEV-Genom } \\
\text { und/oder einer Serokonver- } \\
\text { sion nach der Gabe von Blut- } \\
\text { produkten bei empfangender } \\
\text { Person }{ }^{\text {b }}\end{array}$ \\
\hline & $\begin{array}{l}\text { Empfangende Person war } \\
\text { bereits zum Zeitpunkt der } \\
\text { Gabe von Blutprodukten } \\
\text { HIV-infiziert }\end{array}$ & $\begin{array}{l}\text { Empfangende Person war } \\
\text { bereits zum Zeitpunkt der } \\
\text { Gabe von Blutprodukten } \\
\text { HBV-infiziert }\end{array}$ & $\begin{array}{l}\text { Empfangende Person war } \\
\text { bereits zum Zeitpunkt der } \\
\text { Gabe von Blutprodukten } \\
\text { HCV-infiziert }\end{array}$ & $\begin{array}{l}\text { Empfangende Person war } \\
\text { bereits zum Zeitpunkt der } \\
\text { Gabe von Blutprodukten HEV- } \\
\text { infiziert }\end{array}$ \\
\hline & $\begin{array}{l}\text { Genomsequenzanalyse } \\
\text { schließt phylogenetische } \\
\text { Identität aus (z. B. unter- } \\
\text { schiedlicher Subtyp) }\end{array}$ & $\begin{array}{l}\text { Genomsequenzanalyse } \\
\text { schließt phylogenetische } \\
\text { Identität aus (z. B. unter- } \\
\text { schiedlicher Genotyp) }\end{array}$ & $\begin{array}{l}\text { Genomsequenzanalyse } \\
\text { schließt phylogenetische } \\
\text { Identität aus (z. B. unter- } \\
\text { schiedlicher Genotyp) }\end{array}$ & $\begin{array}{l}\text { Genomsequenzanalyse } \\
\text { schließt phylogenetische Iden- } \\
\text { tität aus (z. B. unterschiedlicher } \\
\text { Genotyp) }\end{array}$ \\
\hline $\begin{array}{l}\text { LoD der ID-NAT-Tests } \\
\text { zum Ausschluss der } \\
\text { Spenderinfektion }\end{array}$ & $\begin{array}{l}\mathrm{NAT} \leq 100 \mathrm{IU} / \mathrm{ml}(\mathrm{HIV}-1 \\
\mathrm{RNA})^{c}\end{array}$ & $\leq 7 \mathrm{IU} / \mathrm{ml}$ & $\leq 20 \mathrm{IU} / \mathrm{ml}$ & $\leq 50 \mathrm{IU} / \mathrm{ml}$ \\
\hline $\begin{array}{l}\text { Zielregion für die } \\
\text { Genomsequenzanalyse } \\
\text { zum Ausschluss der } \\
\text { Virusidentität }\end{array}$ & $\begin{array}{l}\text { Polymerasegen (reverse } \\
\text { Transkriptase, Polymera- } \\
\text { se, Integrase analog der } \\
\text { Resistenztestung) und } \\
\text { zusätzlich, falls es keine } \\
\text { eindeutige Aussage gibt, } \\
\text { noch den V3 Loop (analog } \\
\text { der Korezeptoranalyse) }\end{array}$ & ORF für das S-/Pol-Gen & HVR1-kodierende Region & $\begin{array}{l}\text { ORF1-Gen oder eine hyperva- } \\
\text { riable Zielregion in ORF2 (z. B.: } \\
\text { nt } 5885 \text { bis } 6510 \text { der GenBank- } \\
\text { Sequenz NC001434) }\end{array}$ \\
\hline \multicolumn{5}{|c|}{$\begin{array}{l}\text { 's. Anhänge A2, A3, B2, C2, C3, D } \\
\text { blabordiagnostischer Nachweis entsprechend den AWMF-Leitlinien (HIV, HCV, HBV) bzw. Empfehlungen der Europäischen Vereinigung für das Studium } \\
\text { der Leber (EASL, https://easl.eu/wp-content/uploads/2018/10/EASL-CPG-hepatitis-E-virus-infection.pdf) (HEV) } \\
\text { 'Bei Verdacht auf HIV-2 ist eine hochempfindliche HIV-2 NAT Testung durchzuführen }\end{array}$} \\
\hline
\end{tabular}

